\title{
Atividade física na rotina de indivíduos em tratamento para câncer de próstata em Vitória da Conquista e região
}

Physical activity in routine of individuals in treatment for prostate cancer in Vitória da Conquista and region

\author{
Y. P. Muniz ${ }^{1}$; A. T. Almeida ${ }^{*}$; C. L. Souza ${ }^{2}$; L. Tomazi ${ }^{3}$ \\ ${ }^{1}$ Universidade Federal da Bahia, Instituto Multidisciplinar em Saúde, Campus Anísio Teixeira, 45029-294, Vitória da \\ Conquista, Bahia, Brasil.
}

${ }^{2}$ Laboratório de Análises clínicas, Núcleo Interdisciplinar de Atenção à Saúde, Universidade Federal da Bahia, Instituto Multidisciplinar em Saúde, Campus Anísio Teixeira, 45029-294, Vitória da Conquista, Bahia, Brasil.

${ }^{3}$ Laboratório de Biologia celular e molecular, Núcleo de Biointegração, Universidade Federal da Bahia, Instituto Multidisciplinar em Saúde, Campus Anísio Teixeira, 45029-294, Vitória da Conquista, Bahia, Brasil.

*andreitalmeida85@gmail.com

(Recebido em 24 de julho de 2020; aceito em 18 de janeiro de 2021)

O câncer de Próstata $(\mathrm{CaP})$ é uma neoplasia com vários estágios de evolução e frequentemente associada a homens de idade avançada. O presente trabalho teve o objetivo de analisar fatores associados a prática de atividade física em pacientes diagnosticados com (CaP) sob tratamento no Sistema Único de Saúde (SUS) em Vitória da Conquista (BA). Compuseram esta amostra 150 indivíduos com CaP com mais de 50 anos, atendidos na Unidade de Alta Complexidade em Oncologia (UNACON). Dados foram obtidos através de aplicação de questionário semiestruturado, aferição de medidas antropométricas e coleta de dados em prontuário médico. A análise incluiu estatística descritiva e de associação entre prática de atividade física e $\mathrm{CaP}$ considerando significância quando $\mathrm{p}<0,05$. Dos 150 pacientes, $45,3 \%$ praticavam atividade física, sendo a caminhada a mais frequente. A subamostra de praticantes de atividade física apresentou $85,3 \%$ de indivíduos não brancos com idade média 71 anos $\pm 7,26$. Houve associação entre prática de atividade física e idade inferior a 74 anos ( $p$-value 0,002 ), e autopercepção de saúde boa/muito boa ( $p$-value 0,001$)$. A prática de atividade física é possível na vigência de doenças como câncer; se associando a melhor autopercepção de saúde e tendo o aumento da idade como fator limitante.

Palavras-chave: fator de risco, câncer, Sistema Único de Saúde

Prostate cancer $(\mathrm{CaP})$ is a neoplasm with several stages of evolution and often associated with men of advanced age. The present study aimed to analyze associates factors to practice of physical activity in patients diagnosed with (CaP) under treatment in the Unified Health System (SUS) in Vitória da Conquista (BA). This sample comprised 150 individuals with CaP over 50 years of age, seen at the High Complexity Unit in Oncology (UNACON). Data were obtained by applying a semi-structured questionnaire, measuring anthropometric measurements and collecting data from medical records. Statistical analysis included descriptive statistics, and statistical association between physical activity and explanatory variables, considering significance when $\mathrm{p}<0.05$. Of the 150 patients, $45.3 \%$ practiced physical activity, with walking being the most frequent. The subsample of practitioners of physical activity $85.3 \%$ of non-white individuals with a mean age of 71 years \pm 7.26 . There was an association between physical activity and age below 74 years (p-value 0.002), and self-perception of good / very good health (p-value 0.001 ). The practice of physical activity is possible in the presence of diseases such as cancer; associating with better self-perceived health and with increasing age as a limiting factor.

Keywords: risk factor, cancer, Health Unic System

\section{INTRODUÇÃO}

$\mathrm{O}$ câncer de próstata $(\mathrm{CaP})$ é uma doença maligna comumente ligada ao avanço da idade dos homens [1]. Mundialmente, o CaP representa 14\% de todos os casos incidentes de câncer e $6 \%$ das mortes [2]. No Brasil, constitui a segunda neoplasia mais frequente em homens, perdendo apenas para o câncer de pele não melanoma [1]. 
A incidência de $\mathrm{CaP}$ tem aumentado nas últimas décadas. Estudos estimam que em até 15 anos o CaP será a neoplasia maligna mais comum no mundo [3]. O Instituto Nacional do Câncer (INCA) prevê de risco estimado para o ano de 2020 de 65,8 casos novos a cada 100 mil homens no Brasil, sendo 6.130 mil casos novos para a Bahia [4]. Entretanto, mesmo diante destas estimativas, o CaP possui bom prognóstico, tendo uma probabilidade de sobrevida em cinco anos acima de $80 \%$, variando em função de fatores genéticos, clínicos, socioeconômicos e ambientais [5].

No estágio inicial de desenvolvimento, o CaP é assintomático. Quando atinge estágios mais avançados pode apresentar sinais e sintomas que frequentemente interferem negativamente na qualidade de vida do paciente [6]. Fadiga, dores musculares, cansaço, mudança de peso, nictúria, redução da força e calibre do jato urinário, sensação de não esvaziamento completo da bexiga, incontinência urinária e dores durante a micção estão entre os achados semióticos mais frequentes [3-6].

Apesar do quadro clínico e morbidade presentes em pacientes com CaP, não é raro a prática de atividade física entre eles. Estudos [7-9] têm demonstrado que a prática regular de exercícios físicos moderados por estes pacientes (como caminhada) e principalmente os exercícios vigorosos (como natação, corrida e ciclismo) conduzem a vários benefícios. Dentre os benefícios destacam-se: (i) melhor prognóstico da doença [8]; (ii) retardo da progressão do CaP [8]; (iii) redução do risco de $\mathrm{CaP}$ avançado e de morte decorrente [9]; (iv) redução da mortalidade global [7]; e (v) melhora da resposta aos sinais e sintomas da doença, garantindo melhor qualidade de vida aos pacientes [7] Ainda foi relatado que indivíduos com $\mathrm{CaP}$ que exercem prática moderada de atividade vigorosa por 90 minutos por semana apresentaram risco 33\% menor de morrer por qualquer causa e $35 \%$ menor, devido ao $\mathrm{CaP}$ [7]. Foi demonstrado ainda que a prática superior a 3 horas semanais, inferiram risco $61 \%$ menor de morte pela doença quando comparada com homens que praticavam atividades físicas vigorosas por menos de 1 hora por semana [7, 8].

É sabido que a prática de atividade física em indivíduos saudáveis é benéfica. Porém, tal prática não recebe a devida atenção em pacientes com doenças graves como o câncer. A atividade física pode produzir alterações metabólicas e morfológicas que podem torná-la importante no tratamento adjuvante e recuperação de pacientes com câncer [10]. A prática pode melhorar a capacidade cardiovascular, diminuir gordura corporal e aumentar a resistência muscular além da força e flexibilidade. Estes fatores influenciam na diminuição de alterações deletérias causadas pelo metabolismo e proporcionam melhor expectativa no combate a tumores em geral [11]. Apesar destas associações favoráveis, é perceptível baixo número de estudos voltados sobre atividade física associada ao tratamento do câncer [11], especialmente ao CaP. Cumpre ressaltar ainda que as grandes diferenças de impedimentos e morbidades associadas provocadas pelos diferentes tipos e tumores, seus estágios de desenvolvimento e agressão além dos tratamentos utilizados, reclamam por estudos que sejam delineados especificamente para o estudo da prática de atividade física nos diferentes tipos de tumores considerando este conjunto de variáveis incrementar as evidências sobre o tema.

Diante do contexto apontado e da importância do tema, o objetivo do presente estudo foi analisar fatores associados à prática de atividade física em pacientes portadores de $\mathrm{CaP}$ sob tratamento no Sistema Único de Saúde (SUS) no sudoeste da Bahia.

\section{MATERIAL E MÉTODOS}

\subsection{Desenho experimental e Amostra estudada}

Trata-se de estudo descritivo e exploratório derivado de estudo maior intitulado "Caracterização de amostra e levantamento de variáveis possivelmente relevantes para o câncer de próstata em indivíduos de etnia negra e não-negra de Vitória da Conquista-BA". O estudo maior (tipo casocontrole) incluía aproximadamente 300 indivíduos, sendo 150 do grupo caso em tratamento para CaP pelo SUS na Unidade de Alta Complexidade em Oncologia (UNACON), localizado em Vitória da Conquista - BA. Apenas o grupo caso (150 pacientes de $\mathrm{CaP}$ ) foi utilizado no presente estudo. Os critérios de inclusão para o grupo caso foram: (i) idade $\geq 50$ anos e (ii) diagnóstico anatomopatológico de $\mathrm{CaP}$. 
Os dados foram coletados de julho de 2015 a janeiro de 2016. A participação voluntária dos indivíduos envolveu assinatura de Termo de Consentimento Livre e Esclarecido (TCLE). O projeto foi aprovado pelo Comitê de Ética em Pesquisa da Universidade Federal da Bahia, Instituto Multidisciplinar em Saúde (UFBA-IMS), Protocolo 30879614.3.0000.5556.

\subsection{Coleta de dados}

A coleta de dados incluiu: (i) Aplicação de questionário, (ii) Aferição de medidas antropométricas e (iii) Coleta de dados em prontuários médicos. O questionário foi elaborado e otimizado a partir de instrumento de coleta de dados da Pesquisa Nacional de Saúde Pública [12]. $\mathrm{O}$ instrumento adaptado para o presente estudo abordava oito categorias: (i) Caracterização sociodemográfica; (ii) Hábitos de vida; (iii) Condição de saúde; (iv) Uso dos serviços de saúde; (v) Uso de medicamentos (com exceção dos utilizados no tratamento para o CaP); (vi) Informações de respondente próximo; (vii) Medidas antropométricas; e (viii) Dados do prontuário médico.

A aferição das medidas antropométricas ocorreu por: (i) pesagem em balança digital (Marte ${ }^{\circledR}$, modelo LC200-PP), (ii) medida da altura do indivíduo com estadiômetro vertical (Alturexata ${ }^{\circledR}$ ), e (iii) quando necessário (para indivíduos com curvatura postural) medida da altura do joelho com antropômetro (Indaia ${ }^{\circledR}$ ). Os indivíduos foram pesados e medidos seguindo as recomendações do Ministério da saúde [13-14]. Considerando (i) o critério de inclusão idade $\geq 50$ anos; (ii) o CaP uma doença mais frequente em indivíduos com idade $\geq 65$ anos, o estado nutricional dos indivíduos foi classificado de acordo com o Índice de Massa Corporal (IMC=peso/estatura ${ }^{2}$ ) para idosos. Os indivíduos foram considerados: (i) desnutridos, quando $\operatorname{IMC}\left(<22,0 \mathrm{Kg} / \mathrm{m}^{2}\right)$; (ii) eutróficos, quando IMC entre (22,0 a 27,0 Kg/m²); e (iii) sobrepeso/obesidade quando IMC (> 27,0 Kg/m²) [15]. Idade inferior a 50 anos e a recusa em participar da pesquisa constituíram os critérios de exclusão do estudo.

Informações dos prontuários médicos dos pacientes foram coletadas nas dependências da UNACON. O escore de Gleason, um dos parâmetros analisados neste estudo, se baseia na análise anatomopatológica de tecido prostático, constitui importante indicador prognóstico de CaP. É categorizado em pontuação de 6 a 10 que resulta da soma dos padrões primário e secundário. Escores maiores estão associados a tumores mais agressivos [16].

$\mathrm{O}$ tratamento para $\mathrm{CaP}$ dos pacientes deste estudo foi prioritariamente hormonal, tanto na forma oral quanto injetável. No tratamento oral, usou-se bicalutamida e leuprorrelina. Estes medicamentos, considerados privadores de andrógenos, foram administrados em posologia diária (bicalutamida) ou mensal (leuprorrelina).

\subsection{Análise estatística}

A caracterização e análise descritiva dos pacientes $(\mathrm{n}=150)$ foi realizada em relação à prática de atividade física. Os indivíduos foram considerados praticantes de atividade física quando (no corte temporal da entrevista) responderam que realizavam habitualmente as seguintes atividades: (i) caminhada, (ii) corrida, (iii) andar de bicicleta, (iv) trabalho braçal na roça, (v) trabalho braçal de limpeza de casa, e/ou (vi) "outro" tipo de atividade física.

Foi investigada possível associação da prática de atividade física em pacientes com $\mathrm{CaP}$ com as demais variáveis - consideradas potenciais fatores de risco e/ou de proteção para o $\mathrm{CaP}$ (variáveis resposta). Estas variáveis resposta analisadas foram: (i) idade, (ii) cor autorreferida, (iii) consumo de álcool autorreferido atualmente (no período da aplicação do questionário), (iv) consumo de cigarros autorreferido atualmente, (v) autopercepção de saúde, (vi) histórico de CaP familiar autorreferido, (vii) histórico de DST autorreferido, (viii) uso de medicamentos autorreferido atualmente, (ix) IMC calculado, (x) Escore de Gleason (informações do prontuário) e (xi) hormonioterapia (informações do prontuário).

A posteriori, foram realizados cálculos do poder estatístico para as variáveis independentes avaliadas com resultados de OR significativos. Para tanto, considerou-se $95 \%$ de intervalo de confiança bicaudal. O poder estatístico foi mensurado entre 94,4 e $100 \%$, baseado na aproximação normal com correção continuada. 
Os dados foram compilados em planilha eletrônica no programa Microsoft Excel 2010. A análise dos dados incluiu estatística descritiva para varáveis categóricas e média e desvio-padrão para as variáveis contínuas. Também realizada análise de associação com uso da Odds ratio (OR) para verificação de associação entre a prática de atividade física e as variáveis resposta, adotandose intervalo de confiança (IC) de 95\%. As associações foram consideradas estatisticamente significativas quando valor de $\mathrm{p}<0,05$. As análises dos dados foram realizadas através do programa Epi Info, versão 7.1.5.

\section{RESULTADOS}

Dos pacientes com CaP estudados, 45,3\% ( $\mathrm{n}=68)$ eram praticantes de atividade física, sendo a maioria 78,7\% de não brancos e com média de idade de $74 \pm 8,1$ anos. Dentre as atividades físicas praticadas de forma isolada, as mais citadas foram: (i) caminhada (22,6\%); (ii) trabalho braçal na roça (14\%); (iii) outros (2,6\%); (pedreiro e marceneiro) e (iv) trabalho braçal na limpeza de casa $(0,7 \%)$. A associação da prática de mais de um tipo de atividade física foi baixa $(5,4 \%)$. Destas, a associação mais mencionada foi caminhada e trabalho braçal na roça $(3,3 \%)$.

Os resultados da análise univariada da prática de exercício físico pelos pacientes em tratamento do CaP estão demonstrados na Tabela 1. A caracterização da subamostra dos praticantes de atividade física $(\mathrm{n}=68)$ demonstrou (i) maior frequência de não brancos $(85,3 \%)$ e (ii) média de idade de $71 \pm 7,26$ anos (dados não demonstrados).

Tabela 1. Análise univariada da prática de atividade física por pacientes com CaP em tratamento pelo SUS em Vitória da Conquista (BA), 2016.

\begin{tabular}{|c|c|c|c|c|}
\hline Fatores associados analisados & $\begin{array}{c}N \\
(150)^{l}\end{array}$ & $\begin{array}{l}\text { Prática de } \\
\text { atividade } \\
\text { física } N(\%)\end{array}$ & OR $(I C 95 \%)$ & $\begin{array}{l}\text { Valor de } \\
p^{2}\end{array}$ \\
\hline \multicolumn{5}{|l|}{ Idade } \\
\hline$<74$ & 71 & $41(60,3)$ & $2,63(1,36-5,10)$ & 0,00 \\
\hline$\geq 74$ & 79 & $27(39,7)$ & 1 & \\
\hline \multicolumn{5}{|l|}{ Cor da pele autorreferidas } \\
\hline Não branco & 118 & $53(77,9)$ & $0,92(0,42-2,02)$ & 0,41 \\
\hline Branco & 32 & $15(22,1)$ & 1 & \\
\hline \multicolumn{5}{|c|}{ Consumo de álcool autorreferido atualmente* } \\
\hline Não & 136 & $59(86,8)$ & $2,35(0,75-7,38)$ & 0,07 \\
\hline Sim & 14 & $9(13,2)$ & 1 & \\
\hline \multicolumn{5}{|c|}{ Consumo de cigarro autorreferido atualmente* } \\
\hline Não & 133 & $59(86,8)$ & $1,61(0,57-4,59)$ & 0,19 \\
\hline Sim & 16 & $9(13,2)$ & 1 & \\
\hline \multicolumn{5}{|l|}{ Autopercepção de saúde } \\
\hline Muito boa/ Boa Regular/ruim/muito & 82 & $44(64,7)$ & $2,07(1,07-4,02)$ & 0,01 \\
\hline ruim & 67 & $24(35,3)$ & 1 & \\
\hline \multicolumn{5}{|l|}{ Histórico de CaP familiar autorreferido } \\
\hline Não & 86 & $36(56,3)$ & $1,30(0,66-2,53)$ & 0,22 \\
\hline Sim & 58 & $28(43,7)$ & 1 & \\
\hline \multicolumn{5}{|l|}{ Histórico de DST autorreferido } \\
\hline Não & 116 & $54(79,4)$ & $0,85(0,39-1,85)$ & 0,34 \\
\hline Sim & 33 & $14(20,6)$ & 1 & \\
\hline \multicolumn{5}{|l|}{ Uso de medicamentos autorreferido** } \\
\hline Sim & 115 & $49(72,1)$ & $0,62(0,29-1,33)$ & 0,11 \\
\hline Não & 35 & $19(27,9)$ & 1 & \\
\hline
\end{tabular}


Tabela 1 (continuação)

\begin{tabular}{lcccc}
\hline Fatores associados analisados & $N$ & $\begin{array}{c}\text { Prática de } \\
\text { atividade } \\
\text { física } N(\%)\end{array}$ & OR (IC 95\%) & $\begin{array}{c}\text { Valor } \\
\text { de } p^{2}\end{array}$ \\
\hline $\begin{array}{l}\text { Idade } \\
\text { Desnutrido/eutrófico }\end{array}$ & 101 & $45(67,2)$ & $1,52(0,73-3,17)$ & 0,13 \\
Sobrepeso & 40 & $22(32,8)$ & 1 & \\
Escore de Gleason & & & & \\
$\leq 7$ & 114 & $53(77,9)$ & $0,90(0,42-1,96)$ & 0,43 \\
$\geq 8$ & 34 & $15(22,1)$ & 1 & \\
Hormonioterapia & & & & \\
$\leq 8$ & 35 & $14(20,6)$ & $1,33(0,61-2,86)$ & 0,24 \\
$\geq 9$ & 115 & $54(79,4)$ & 1 & \\
\hline
\end{tabular}

Nota: Dados coletados na UNACON, de 07/2015 a 01/2016. Todos os dados aqui mencionados foram coletados através da aplicação de questionário, com exceção de IMC calculado, escore de Gleason e Hormonioterapia. As informações destas duas últimas variáveis foram coletadas de prontuários. Não praticantes de atividade física $=82 . *$ Atualmente $=$ no período de aplicação do questionário. ** Refere-se aos medicamentos utilizados, com exceção dos empregados no tratamento para o CaP. ${ }^{1}$ Totais variam devido a informações ignoradas. ${ }^{2}$ Significância estatística adotada $\mathrm{p}<0,05$.

Houve maior frequência da prática em pacientes com idades inferiores a 74 anos $(60,3 \%)$. Os resultados apontaram 2,63 (1,36 - 5,10) vezes a chance de praticar atividade física quando comparado aqueles com idade $\geq 74$ anos; $p$-value 0,002 . Sobre o consumo de bebida alcóolica a maioria dos praticantes de atividade física relatou não consumir bebida alcóolica $(86,8 \%)$. Os dados indicam que estes pacientes apresentaram $2,35(0,75-7,38)$ vezes a chance de praticar atividade física em relação àqueles que bebem, $p$-value 0,07 ; valor próximo à significância estatística. Analisando a autopercepção de saúde por parte dos pacientes praticantes de atividade física, houve maior frequência dos que consideravam sua saúde "Muito boa/Boa" $(64,7 \%)$. Os dados sugerem que estes pacientes possuíram 2,07 $(1,07-4,02)$ vezes a chance de praticar atividade física em relação quem àqueles que se autopercebiam com saúde "Regular/ruim/muito ruim", $p$-value 0,01 .

\section{DISCUSSÃO}

Atualmente, o tratamento dos pacientes com CaP é baseado em cirurgia, radioterapia e terapia farmacológica, que habitualmente incluem muitos efeitos adversos. Como forma de amenizar esse quadro, medidas de intervenção não farmacológicas têm sido agregadas como estratégia terapêutica. A prática regular de exercícios físicos tem demonstrado melhorar a qualidade de vida de pacientes com CaP [17]. Dentre os fatores que têm sido influenciados positivamente pela atividade física, ressaltam-se a vitalidade (ausência de fadiga) e desempenho fisiológico [17]. Estes dados sustentam a indicação de prática de atividade física como complementar à terapia desta doença [17].

Neste estudo, no grupo de pacientes com $\mathrm{CaP}, 45,3 \%$ afirmaram ser praticantes de atividade física (média idade $=71$ anos). $O$ que pode ser reflexo do crescente aumento da expectativa de vida dos pacientes com CaP. Estudo em 2014 demonstrou que tais pacientes possuíam taxa de sobrevivência de 99\% em 10 anos pós diagnóstico [18]. Estudo de câncer de mama em mulheres, com média de idade de 52,7 anos, apresentou 76,6\% das pacientes como praticantes de atividade física [19]. É provável que as médias de idades distintas entre os estudos mencionados (das mulheres com câncer de mama e dos homens com $\mathrm{CaP}$ do presente estudo) possam ter influenciado na comparação devido as pacientes com câncer de mama serem, na média, mais jovens e por isso mais aptas à prática. Adicione-se a isto, o fato de que geralmente as mulheres cuidam mais de sua saúde (o que inclui em maior busca pelos serviços de saúde - 2 vezes que os homens) [20]. Observase que, apesar dos tipos de câncer envolvidos nos estudos mencionados apresentarem tratamentos distintos, a prática de atividade física tem se demonstrado útil como complemento dos seus tratamentos. 
No presente trabalho, foi observado que o grupo com idade inferior a 74 anos praticava mais atividade física $(60,3 \%)$ em relação ao grupo mais idoso. O grupo de menor idade apresentou 2,63 vezes a chance de praticar atividade física em relação aqueles com idade $\geq 74$ anos, o que foi estatisticamente significante. $\mathrm{O}$ envelhecimento pode provocar uma série de mudanças como o surgimento de doenças crônicas, perdas cognitivas, sintomas depressivos, declínio sensorial, acidentes, isolamento social e limitações físicas [21]. Estes fatores provocam cansaço e podem conduzir ao estabelecimento de quadro de sedentarismo, além de doenças associadas ao processo de senilidade [22]. Assim, a idade mais avançada e presença do câncer, podem explicar a maior frequência de atividade física no grupo de pacientes com menor idade. Em estudo sobre associação de atividade física com mortalidade por todas as causas entre adultos mais velhos, verificou-se associação inversa entre mortalidade e câncer; além de relato que os pacientes se beneficiaram com estilo de vida ativo [23].

No que se refere ao consumo de álcool, dentre os pacientes praticantes de atividade física, houve uma relação inversa entre uso de álcool e atividade física. Aqueles que não bebiam tiveram 2,35 a chance de praticar atividade física em relação aos que consumem álcool, $p$-value de 0,07. Embora não houvesse significância ao nível de $95 \%$, estudos têm mostrado relação inversa entre consumo de álcool e prática de atividade física [24]. Entende-se que o tamanho amostral limitado do presente estudo, imputou limitação na avaliação de algumas associações. Em pesquisa realizada com pacientes portadoras de câncer de mama apresentando relato de 38,6\% de pacientes praticantes de atividade física, observou-se que $82,4 \%$ eram não etilistas [25]. Ambos os estudos aqui abordados se assemelham na tendência de não ingestão de bebida alcóolica por praticantes de atividade física.

Quanto ao consumo de cigarro em pacientes praticantes de atividade física, observou-se maior frequência de indivíduos que não fumavam atualmente $(86,8 \%)$. Em estudo de pacientes com câncer colorretal (em fase pós-operatória) com média de idade de 58,3 anos, praticantes de atividade física que não fumavam atualmente representaram 68,6\% da amostra [26]. O presente estudo concorda com estas pesquisas relatando também elevada presença de praticantes de atividade física que optaram por fumar menos ou não fumar ainda que sejam tumores de outras origens. Estes dados permitem apontar provável relação entre prática de atividade física e menor consumo de cigarros. Embora neste estudo não tenha sido verificada associação significativa (OR $1,61 p$-value 0,19$)$.

A variável autopercepção de saúde "Muito boa/Boa" indicou 2,07 vezes a chance de praticar atividade física em relação aos que autopercebiam sua saúde como "Regular/ruim/muito ruim" com $p$-valor $=0,01$. Pesquisa realizada no Rio Grande do Sul sobre autopercepção da saúde em idosos, com maior frequência de mulheres $(63,13 \%)$ e idades entre 60 a 69 anos, verificou que $67,52 \%$ praticavam algum tipo de atividade física e $61,33 \%$ apresentaram autopercepção da saúde boa/muito boa [27]. Se compararmos a frequência de praticantes de atividade física dos pacientes com CaP (do presente estudo - 45,3\%) com a encontrada no estudo mencionado anteriormente $(67,52 \%)$ [27], percebe-se que há diferença de mais de $20 \%$ entre elas. Isso pode ser reflexo do diagnóstico do câncer ainda ser considerado um estigma para os homens. No imaginário coletivo, a doença é frequentemente associada a morte, e este fato pode influenciar na alteração de suas dimensões físicas, psíquicas e sociais [28]. Entretanto, o tratamento do $\mathrm{CaP}$ ocorre de forma individualizada, levando em conta estágio do tumor, idade, tamanho da próstata, grau histológico, comorbidades, expectativa de vida, anseios do paciente e recursos técnicos disponíveis [29]. Percebe-se que, na fase inicial (passível de tratamento curativo) os pacientes com CaP não desenvolvem qualquer sinal ou sintoma relacionado à doença, possibilitando aos pacientes menos problemas físicos e manutenção de maior autonomia sobre seu corpo [30]. Ressalta-se que não foram encontrados estudos relacionando autopercepção da saúde com prática de atividade física em indivíduos com CaP. Todavia, a própria prática de atividade física, por si só, tem sido relacionada a autopercepção positiva da saúde [31].

Em relação ao uso de medicamentos (excetuando-se o tratamento para o $\mathrm{CaP}$ ) nos pacientes praticantes de atividade física com $\mathrm{CaP}$, verificou-se achados de elevada frequência de uso de medicamentos $(72,1 \%)$. Considerando-se se tratar de grupo de idosos, compreende-se esta causalidade. O câncer é uma das doenças crônicas não transmissíveis que mais contribui para o aumento da morbimortalidade [32]. É esperado que o paciente com CaP, associado ao processo de envelhecimento, fique mais susceptível a outras comorbidades, e aumente o consumo de 
medicamentos. Entretanto, pesquisas vêm associando a prática de atividade física como um fator amenizador das limitações provocadas pelo envelhecimento [33]. Neste sentido, mesmo não tendo sido determinado os efeitos diretos da atividade física sobre o uso de medicamentos na população idosa, acredita-se, que além dos benefícios físicos proporcionados, possa gerar também diminuição no uso de medicamentos [33].

Foi verificada maior frequência de indivíduos com desnutrição/eutrofia $(67,2 \%)$ no grupo de praticantes de atividade física. Destes, 53,8\% eram eutróficos (dados não mostrados na Tabela 1). Em estudo de câncer colorretal, verificou-se que dentre praticantes de exercício físico 54,93\% eutróficos [34]. Assim como ocorre com o CaP, a detecção e tratamento precoce de pacientes com câncer colorretal elevaram as taxas de sobrevivência [35]. Assim, compreende-se a maior frequência de pacientes com câncer colorretal, aderentes à prática de atividade física, proporcionando um perfil mais eutrófico [34]. Destaca-se que alguns estudos avaliam que a prática de atividade física, se bem estruturada e orientada, pode ajudar a atingir e/ou manter a eutrofia [3637]. Esta associação tem contribuído para controle e prevenção de fatores de risco de doenças coronárias como o perfil lipídico, resistência à insulina, diabetes e hipertensão. Ao comparar os trabalhos mencionados, foi possível observar que as frequências de eutrofia entre pacientes praticantes de atividade física em tratamento para $\mathrm{CaP}$ e colorretal são semelhantes. É provável que no presente trabalho tenha ocorrido subestimação da associação de alterações do IMC com a prática de exercício físico, devido à amostra pequena.

O Escore de Gleason em pacientes praticantes de atividade física do presente estudo, associouse à escores $\leq 7(77,9 \%)$ corroborado por estudo semelhante [7]. Escores de Gleason $\geq 7$ são associados a tumores mais agressivos, invasão capsular e extraprostática [38] e maior morbidade. É esperado que a pratica de atividade física seja inversamente proporcional ao Escore de Gleason.

Em relação a hormonioterapia, os pacientes praticantes de atividade física estiveram entre os que se submeteram a 9 ou mais aplicações de hormônio injetável $(79,4 \%)$. O que indica que os pacientes com $\mathrm{CaP}$ que compuseram a amostra estudada não eram virgens de tratamento, e seguiam com regularidade o tratamento. Estudos analisando atividade física (no lazer) e melhoria da qualidade de vida de pacientes com $\mathrm{CaP}$, observaram que pacientes tratados à base de terapia de privação de andrógenos (ADT), eram significativamente menos ativos quando comparados aos tratados apenas com radioterapia [39]. Estudo sobre atividade física praticada por pacientes com $\mathrm{CaP}$ que recebiam $\mathrm{ADT}$ (benefícios de intervenção de 16 semanas), mostraram que a prática atenua efeitos colaterais da ADT [40].

\section{CONCLUSÃO}

É notória a contínua busca por estratégias terapêuticas que adicionem efetividade ao tratamento, conforto e qualidade de vida aos pacientes com CaP. Dentro desta perspectiva, foi demonstrada associação entre prática de atividade física, e idade inferior a 74 anos com autopercepção positiva do estado de saúde. Tal associação se deve ao fato da limitação da prática de atividade física por indivíduos com idade mais avançada, e também pela correlação da prática de exercício com boa percepção de saúde.

Estudos demonstram melhoria na qualidade de vida dos pacientes de câncer [39-40] o que pode incluí-la como terapêutica complementar no tratamento do CaP. Outros estudos [41-42] também aconselham a prática de atividade física para pacientes com $\mathrm{CaP}$ por aliviarem os efeitos colaterais do tratamento, melhorar a qualidade de vida, minimizar comportamento sedentário, diminuir a fadiga, melhorar função sexual, além de diminuição da circunferência abdominal e pressão arterial.

Diante das crescentes evidências da pratica de atividade física por pacientes com $\mathrm{CaP}$ serem benéficas, evidencia-se a necessidade da realização de mais estudos abordando o tema, especialmente ao $\mathrm{CaP}$ com amostragem robusta e desenho prospectivo de acompanhamento a fim de fundamentar melhor o tema e propor efetivo esclarecimento acerca dos fatores associados à prática de atividade física em diferentes populações e nos diferentes tipos de tumores. Cumpre ressaltar ainda que as diferentes limitações proporcionadas pelos tumores e pelas abordagens terapêuticas associadas, podem produzir diferentes impactos na prática de atividades físicas, devido 
aos níveis de morbidade associado. Assim, o estudo da atividade física no câncer deve ser considerado nos diferentes tumores, considerando estágio da doença e tratamentos realizados.

\section{AGRADECIMENTOS}

À Universidade Federal da Bahia (UFBA), por incentivar e promover a realização da presente pesquisa. À Fundação de Amparo à Pesquisa do Estado da Bahia (FAPESB) e ao Programa Institucional de Bolsas de Iniciação Científica (PIBIC), pelo suporte financeiro diante de concessão de bolsas de estudo. A Unidade de Alta Complexidade em Oncologia (UNACON), pela liberação do campo para coleta de dados dos pacientes que compuseram a amostra estudada, e em especial a Ana Cecília Lemos Ferraz pelo apoio logístico. Aos alunos da UFBA Luma Cunha Freitas de Souza, Ítalo Paulino Santana, Douglas da Silva Oliveira, Zilma Alves Santos, Cleriane Santos Macedo e Thamara Louisy Santos Brito pelo auxilio como coletadores de dados de campo.

\section{REFERÊNCIAS BIBLIOGRÁFICAS}

1. Lima RB, Hahn GV. Câncer de próstata e sua relação com a sexualidade masculina: produção científica brasileira. Destaques Acadêmicos. 2016;8(3):70-86, doi: 10.22410/issn.2176-3070.v8i3a2016.1165

2. Lopes A, Chammas R, Iyeyasu H. Oncologia para a graduação. 3rd ed. São Paulo: Lemar; 2013.

3. Rhoden EL, Averbeck MA. Câncer de próstata localizado. Revista da AMRIGS. 2010;54(1):92-9.

4. Instituto Nacional de Câncer José Alencar Gomes da Silva (INCA). Incidência de Câncer de Próstata no Brasil [Internet]. 2020 [acesso em 1 ago 2020]; Disponível em: https://www.inca.gov.br/estimativa

5. Howlander N, Noone AM, Krapcho M, et al., editores. SEER Cancer Statistics Review, 1975-2014. Bethesda: National Cancer Institute; 2017. Disponível em: https://seer.cancer.gov/archive/csr/1975_2014/

6. Goncalves IR; Padovani C, Popim RC. Caracterização epidemiológica e demográfica de homens com câncer de próstata. Ciência Saúde Coletiva. 2008;13(4):1337-42, doi: 10.1590/S141381232008000400031

7. Kenfield SA, Stampfer MJ, Giovannucci EL, et al. Physical activity and survival after prostate cancer diagnosis in the health professionals follow-up study. Journal of Clinical Oncology. 2011;29(6):726-32, doi: $10.1200 / \mathrm{JCO} .2010 .31 .5226$

8. Giovannucci EL, Liu Y, Leitzmann MF, et al. A prospective study of physical activity and incident and fatal prostate cancer. Archives of Internal Medicine. 2005;165(9):1005-10, doi: 10.1001/archinte.165.9.1005

9. Nilsen TI, Romundstad PR, Vatten LJ. Recreational physical activity and risk of prostate cancer: A prospective population-based study in Norway (the HUNT study). International Journal of Cancer. 2006;119(12):2943-7, doi: 10.1002/ijc.22184

10. Keul J. Correlation between physical performance and fatigue in cancer patients. Annals Oncology. 1997;8(12):1251-5, doi: 10.1023/a:1008234310474

11. Battaglini CL, Bottaro M, Campbell JS, et al. Atividade física e níveis de fadiga em pacientes portadores de câncer. Revista Brasileira de Medicina no Esporte. 2004;10(2):98-104, doi: 10.1590/S151786922004000200004

12. Instituto Brasileiro de Geografia e Estatística (IBGE). Pesquisa Nacional de Saúde 2013: Percepção do estado de saúde, estilos de vida e doenças crônicas: Brasil, grandes regiões e unidades da federação. Rio de Janeiro (RJ): IBGE; 2014. Disponível em: http://www.dive.sc.gov.br/conteudos/agravos/publicacoes/PNS\%202013\%20Percep\%C3\%A7\%C3\%A3 o\%20do\%20estado\%20de\%20sa\%C3\%BAde\%20estilos\%20de\%20vida\%20e\%20doen\%C3\%A7as\%2 $0 \mathrm{cr} \% \mathrm{C} 3 \%$ B4nicas.pdf

13. Brasil, Ministério da Saúde. Manual de Antropometria. 2004 [acesso em 1 ago 2020]. Disponível em: http://www.pns.icict.fiocruz.br/arquivos/Manual\%20de\%20Antropometria\%20da\%20PENSE.pdf

14. Brasil, Ministério da Saúde. Manual orientador para aquisição de equipamentos antropométricos. 2012 $\begin{array}{llllll}\text { acesso } & \mathrm{em} & 1 & \text { ago } & 2020] & \text { Disponível }\end{array}$ http://189.28.128.100/dab/docs/portaldab/publicacoes/manual_equipamentos_2012_1201.pdf

15. Lipschitz DA. Screening for nutritional status in the elderly. Primary Care. 1994;21(1):55-67.

16. Magnabosco WJ. Câncer de próstata - diagnóstico e estadiamento. Revista Onco\&. 2014 Jan/Fev;(21):2225. 
17. Gerritsen JKW, Vincent AJP. Exercise improves quality of life in patients with cancer: a systematic review and meta-analysis of randomised controlled trials. British Journal of Sports Medicine. 2016;796803, doi: 10.1136/bjsports-2015-094787

18. Magbanua MJ, Richman EL, Sosa EV, et al. Physical activity and prostate gene expression in men with low-risk prostate cancer. Cancer Causes \& Control. 2014;25(4):515-23, doi: 10.1007/s10552-014-0354$\mathrm{x}$

19. Prado MAS, Mamede MV, Almeida AM, et al. A prática da atividade física em mulheres submetidas à cirurgia por câncer de mama: percepção de barreiras e benefícios. Revista Latino Americana de Enfermagem. 2014;12(3):494-502, doi: 10.1590/S0104-11692004000300007

20. Levorato CD, Mello LM, Silva AS, et al. Fatores associados à procura por serviços de saúde numa perspectiva relacional de gênero. Ciência \& Saúde Coletiva. 2014;19(4):1263-1274, doi: 10.1590/141381232014194.01242013

21. Heck $\mathrm{P}$, Araújo CCR, Pazin J, et al. Disfunção erétil associada à pratica de atividade física e qualidade de vida dos idosos. Revista Brasileira de Atividade Física \& Saúde. 2016;21(2):190-7, doi: 10.12820/rbafs.v.21n2p190-197

22. Stumm EMF, Scherer JA, Kirchner RM, et al. Vivências de idosos submetidos à prostatectomia por câncer: subsídios para o cuidado de enfermagem. Textos \& Contextos. 2010;9(1):89-102.

23. Wu CY, Hu HY, Chou YC, et al. The association of physical activity with all-cause, cardiovascular, and cancer mortalities among older adults. Preventive Medicine. 2015;72:23-9, doi: 10.1016/j.ypmed.2014.12.023

24. Costa JSD, Silveira MF, Gazelle FK, et al. Consumo abusivo de álcool e fatores associados: estudo de base populacional. Revista de Saúde Pública. 2004;38(2):284-91, doi: 10.1590/S003489102004000200019

25. Binotto M, Daltoé T, Formolo F, et al. Atividade física e seus benefícios na qualidade de vida de mulheres com câncer de mama: um estudo transversal em Caxias do Sul - RS. Revista Brasileira de Atividade Física \& Saúde. 2016;21(2):154-61, doi: 10.12820/rbafs.v.21n2p154-161

26. Fortes RC, Recôva VL, Melo AL, et al. Hábitos dietéticos de pacientes com câncer colorretal em fase pós-operatória. Revista Brasileira de Cancerologia. 2007;3(53):277-89.

27. Borges AM, Santos G, Kummer JA, et al. Autopercepção de saúde em idosos residentes em um município do interior do Rio Grande do Sul. Revista Brasileira de Geriatria e Gerontologia. 2014;17(1):79-86, doi: 10.1590/S1809-98232014000100009

28. Moscheta MS, Santos MA. Grupos de apoio para homens com câncer de próstata: revisão integrativa da literatura. Ciência \& Saúde Coletiva. 2012;17(5):1225-33, doi: 10.1590/S1413-81232012000500016

29. Brasil, Ministério da Saúde, Secretaria de Assistência à Saúde, Instituto Nacional de Câncer. Programa Nacional de Controle do Câncer da Próstata: Documento de consenso. Rio de Janeiro (RJ): INCA; 2002. Disponível em: http://bvsms.saude.gov.br/bvs/publicacoes/cancer_da_prostata.pdf

30. Damião R, Figueiredo RT, Dornas MC, et al. Câncer de próstata. Revista HUPE. 2015;14(Supl 1):80-6, doi: 10.12957/rhupe.2015.17931

31. Ferrari RFR, Baldissera VDA, Lange C, et al. Atitude do idoso da comunidade frente ao lazer: Uma interface com a promoção de saúde. Texto \& Contexto - Enfermagem. 2016;25(4): e1280015, doi: 10.1590/0104-07072016001280015

32. Ferraz CCB. Caracterização da capacidade funcional de idosos com câncer de próstata em Mato Grosso do Sul [dissertação]. Campo Grande (MS): Universidade Federal de Mato Grosso do Sul; 2015. 69 p.

33. Souza F. Efeito da atividade física nas condições de saúde, no uso de medicamentos e na qualidade de vida de idosos [dissertação]. Tubarão (SC): Universidade do Sul de Santa Catarina; 2015. 68 p.

34. Arem H, Pfeiffer RM, Engels EA, et al. Pre- and postdiagnosis physical activity, television viewing, and mortality among patients with colorectal cancer in The National Institutes of Health-AARP Diet and Health Study. Journal of Clinical Oncology. 2015;33(2):180-8, doi: 10.1200/JCO.2014.58.1355

35. Hagar FA. Boushey RP. Colorectal cancer epidemiology: Incidence, mortality, survival, and risk factors. Clin Colon Rectal Surg. 2009;22:191-7, doi: 10.1055/s-0029-1242458

36. Barbosa JAS. Estudo sobre o nível de participação, num programa de atividade física e saúde e suas relações com as doenças crônicas não transmissíveis e a qualidade de vida: um estudo de caso [tese]. Campinas (SP): Universidade Estadual de Campinas; 2003. 159 p.

37. Barbosa JAS, Bankoff ADP. Estudo do nível de participação num programa de atividade física e suas relações com as doenças crônicas não transmissíveis. Revista Movimento \& Percepção. 2008;9(12):197220.

38. Cambruzzi E, Zettler CG, Pegas KL, et al. Relação entre escore de Gleason e fatores prognósticos no adenocarcinoma acinar de próstata. Jornal Brasileiro de Patologia e Medicina Laboratorial. 2010;46(1):61-8, doi: 10.1590/S1676-24442010000100011 
39. Chipperfield K, Fletcher J, Millar J, et al. Factors associated with adherence to physical activity guidelines in patients with prostate cancer. Psycho-Oncology. 2013;22(11):2478-86, doi: 10.1002/pon.3310

40. Culos-Reed SN, Robinson JW, Lau H, et al. Physical activity for men receiving androgen deprivation therapy for prostate cancer: benefits from a 16-week intervention. Supportive Care in Cancer. 2010;18(5):591-9, doi: 10.1007/s00520-009-0694-3

41. Friedenreich CM, Wang Q, Neilson HK, et al. Physical activity and survival after prostate cancer. European Urology. 2016;70(4):576-85, doi: 10.1016/j.eururo.2015.12.032

42. Boing L, Seemann T, Souza MC, et al. Benefícios da atividade física em homens com câncer de próstata - Revisão sistemática. Journal of Physical Education. 2016;27:e2729, doi: 10.4025/jphyseduc.v27i1.2729 